\title{
Carleman estimates and controllability results for the one-dimensional heat equation with $B V$ coefficients
}

\author{
Jérôme Le Rousseau \\ Laboratoire d'Analyse Topologie Probabilités, CNRS UMR 6632, Université d'Aix-Marseille I
}

\begin{abstract}
We derive global Carleman estimates for one-dimensional linear parabolic operators $\partial_{t} \pm \partial_{x}\left(c \partial_{x}\right)$ with a coefficient $c$ with bounded variations. These estimates are obtained by approximating $c$ by piecewise regular coefficients, $c_{\varepsilon}$, and passing to the limit in the Carleman estimates associated to the operators defined with $c_{\varepsilon}$. Such estimates yield results of controllability to the trajectories for a classe of semilinear parabolic equations. To cite this article: J. Le Rousseau, C. R. Acad. Sci. Paris, Ser. I(一).

Résumé

Inégalités de Carleman et résultats de contrôle pour une équation de la chaleur à coefficient $\boldsymbol{B V}$ en dimension un. On obtient des inégalités de Carleman pour des opérateurs paraboliques linéaires $\partial_{t} \pm \partial_{x}\left(c \partial_{x}\right)$ avec un coefficient $c$ à variations bornées. Ces inégalités sont obtenues par approximations de $c$ par des coefficients réguliers par morceaux, $c_{\varepsilon}$, et en passant à la limite dans les inégalités de Carleman associées aux opérateurs définis par $c_{\varepsilon}$. De telles inégalités donnent des résultats de contrôlabilité aux trajectoires pour une classe d'équations semilinéaires paraboliques. Pour citer cet article : J. Le Rousseau, C. R. Acad. Sci. Paris, Ser. I-(一).
\end{abstract}

\section{Version française abrégée}

On considère les opérateurs $\partial_{t} \pm A$ dans $Q=(0, T) \times \Omega$, avec $T>0$ et $\Omega=(0,1)$. L'opérateur $A$ est défini par $-\partial_{x}\left(c \partial_{x}\right)$ sur $L^{2}(\Omega)$, où $c$ est à variations bornées $(B V)$ et vérifie $0<c_{\min } \leq c \leq c_{\text {max }}$. Le domaine de $A$ est $D(A)=\left\{u \in H_{0}^{1}(\Omega) ; c \partial_{x} u \in H^{1}(\Omega)\right\}$. On prouve l'inégalité de Carleman globale suivante.

Théorème 0.1 Soit $c \in B V(\Omega)$, avec $0<c_{\text {min }} \leq c \leq c_{\text {max }}$. Soit $\boldsymbol{\aleph}$ l'ensemble des solutions (faibles) de $\partial_{t} q \pm$ $\partial_{x}\left(c \partial_{x} q\right)=f$ dans $Q, q=0 \operatorname{sur}(0, T) \times\{0,1\}, q(T, x)=q_{0}(x)\left(\operatorname{resp} . q(0, x)=q_{0}(x)\right)$ dans $\Omega$, avec $q_{0} \in L^{2}(\Omega)$ et $f \in L^{2}(Q)$.

(i) $\exists \lambda_{1}>0, s_{1}>0$ et $C>0$ tels que l'inégalité de Carleman (4) soit valable pour $s \geq s_{1}, \lambda \geq \lambda_{1}$ et pour tout $q \in \mathbf{N}$.

Email address: jlerous@cmi .univ-mrs.fr (Jérôme Le Rousseau). 
(ii) Si c de est de classe $\mathscr{C}^{1}$ sur $O \Subset \Omega$, avec $O$ un ouvert non vide de $\Omega$, alors on a une inégalité de Carleman avec une 'observation' $\operatorname{sur}(0, T) \times O$.

On donne une idée de la preuve du Théorème 0.1 dans le cas (i), i.e. une 'observation' frontière. Si $c$ est $\mathscr{C}^{1}$ par morceaux, une inégalité de Carleman (4) est obtenue [1,2] en construisant des fonctions poids données dans (3), à partir d'une fonction $\widetilde{\beta}$, régulière par morceaux, qui à chaque point de discontinuité de $c$ satisfait la condition (2).

Dans le cas où $c \in B V(\Omega)$, pour $\varepsilon>0$ on choisit $c_{\varepsilon}$, constant par morceaux, de manière à avoir $\left\|c-c_{\varepsilon}\right\|_{L^{\infty}(\Omega)} \leq \varepsilon$, $V_{0}^{1}\left(c_{\varepsilon}\right) \leq V_{0}^{1}(c)$, où $V_{a}^{b}(\rho)$ est la variation totale de $\rho \operatorname{sur}(a, b)$ [3]. On construit des fonctions $\widetilde{\beta}_{\varepsilon}$ adaptées à $c_{\varepsilon}$ : posant $[\rho]_{x}:=\rho\left(x^{+}\right)-\rho\left(x^{-}\right)$, on exhibe une fonction $f$ telle que i) si $\left[\widetilde{\beta_{\varepsilon}^{\prime}}\right]_{a}=f\left(\left[c_{\varepsilon}\right]_{a}\right)$ en chaque point de discontinuité $a$ de $c_{\varepsilon}$, alors $\widetilde{\beta}_{\varepsilon}$ satisfait la condition (2); ii) de plus $V_{0}^{1}\left(\widetilde{\beta}_{\varepsilon}^{\prime}\right)$ est majorée uniformément par rapport à $\varepsilon$. À une sous-suite près, ces fonctions $\widetilde{\beta}_{\varepsilon}^{\prime}$ convergent partout quand $\varepsilon$ tend vers 0 , par le théorème de Helly. On déduit, par passage à la limite et intégration, une fonction $\widetilde{\beta}$.

À l'aide de $\widetilde{\beta}_{\varepsilon}$ et $\widetilde{\beta}$, on construit des fonctions poids selon (3). Celles associées à $c_{\varepsilon}$ permettent d'obtenir des inégalités de Carleman pour $\partial_{t} \pm \partial_{x}\left(c_{\varepsilon} \partial_{x}\right)$. Une inspection détaillée de la preuve des inégalités de Carleman dans le cas $\mathscr{C}^{1}$ par morceaux permet de prouver l'existence d'une borne uniforme, par rapport à $\varepsilon$, pour la constante $C$ à droite de (4) pour les opérateurs $\partial_{t} \pm \partial_{x}\left(c_{\varepsilon} \partial_{x}\right)$. De même, les constantes $s_{1}$ et $\lambda_{1}$ dans le Théorème 2.2 peuvent être choisies uniformes par rapport à $\varepsilon$. Ces résultats permettent un passage à la limite dans les inégalités de Carleman associées à $\partial_{t} \pm \partial_{x}\left(c_{\varepsilon} \partial_{x}\right)$ : les solutions de $\partial_{t} q_{\varepsilon} \pm \partial_{x}\left(c_{\varepsilon} \partial_{x}\right) q_{\varepsilon}=f$ sont telles que $\partial_{t} q_{\varepsilon}, q_{\varepsilon}, \partial_{x} q_{\varepsilon}, \partial_{x}\left(c \partial_{x} q_{\varepsilon}\right)$ convergent vers les fonctions associées pour la solution $q$ de $\partial_{t} q \pm \partial_{x}\left(c \partial_{x}\right) q=f$, pour $f$ régulière et les conditions finales (resp. initiales) bien choisies; les fonctions poids convergent et les constantes restent bornées. On relaxe alors la régularité sur $f$ et les hypothèses sur les conditions finales (resp. initiales).

Le Théorème 0.1 permet d'obtenir des résultats de contrôlabilité aux trajectoires pour une classe d'équations semilinéaires paraboliques. Si $\mathscr{G}(x, y)=x g(x, y)+y G(x, y)$, avec $g$ et $G$ dans $L_{\text {loc }}^{\infty}(\mathbb{R})$.

Théorème 0.2 Soit $T>0$. Sous les hypothèses du Théorème 0.1, on a :

(i) Contrôlabilité à zéro locale : Il existe $\varepsilon>0$ tel que pour tout $y_{0}$ dans $L^{2}(\Omega)$ avec $\left\|y_{0}\right\|_{L^{2}(\Omega)} \leq \varepsilon$, il existe un contrôle $v \in L^{\infty}(0, T)$ tel que la solution de (1) satisfasse $y(T)=0$.

(ii) Contrôlabilité à zéro globale : Si $\mathscr{G}$ satisfait de plus (7), alors pour tout $y_{0} \in L^{2}(\Omega)$, il existe $v \in L^{\infty}(0, T)$ tel que la solution de (1) satisfasse $y(T)=0$.

On obtient des résultats similaires pour le système (6) avec un ouvert non vide $\omega \Subset \Omega$, et $v \in L^{\infty}((0, T) \times \omega)$, en supposant une régularité $\mathscr{C}^{1}$ du coefficient c sur une partie ouverte non vide de $\omega$.

Le preuve du Théorème 0.2 repose sur un argument de point fixe $[4,5,6]$.

\section{Introduction}

We consider the elliptic operator $A$ formally defined by $-\partial_{x}\left(c \partial_{x}\right)$ on $L^{2}(\Omega)$ in the one-dimensional bounded domain $\Omega=(0,1)$, with $0<c_{\text {min }} \leq c \leq c_{\text {max }}$, and $c$ is assumed to be of bounded variations $(B V)$. The domain of $A$ is $D(A)=\left\{u \in H_{0}^{1}(\Omega) ; c \partial_{x} u \in H^{1}(\Omega)\right\}$. Let $T>0$. We shall use the following notations $Q=(0, T) \times \Omega, \Gamma=\{0,1\}$, and $\Sigma=(0, T) \times \Gamma$.

Here, we show that we can achieve global Carleman estimates for the operators $\partial_{t} \pm A$ in $Q$, with a boundary 'observation' or an interior 'observation'. With such Carleman estimates at hand, we treat the problem of the controllability to the trajectories for semilinear parabolic systems of the form

$$
\begin{cases}\partial_{t} y-\partial_{x}\left(c \partial_{x} y\right)+\mathscr{G}\left(y, \partial_{x} y\right)=0 & \text { in } Q \\ y(t, 0)=v(t), y(t, 1)=0, & \text { in } \Omega \\ y(0, x)=y_{0}(x) & \end{cases}
$$


where $v$ is the control, $\mathscr{G}: \mathbb{R}^{2} \rightarrow \mathbb{R}$ is locally Lipschitz and $\mathscr{G}(0,0)=0$ (further assumptions on the nonlinear function $\mathscr{G}$ or on the initial condition will be introduced below).

A null controllability result for a linear parabolic equation with $B V$ coefficients was proven in [7]. The proof relies on Russell's method [8]. However, the question of the existence of a Carleman-type observability estimate was open. In a recent work [9], a result of controllability for the one-dimensional heat equation with general bounded coefficients was proved. The method of [9] is based on that of [10]. However, with such a method, the knowledge of the observability constant is not sufficient to address the controllability of non-linear heat equations. Carleman estimates, of the form proved here, provide a more precise knowledge of this constant with respect to lower-order terms in the partial differential operator, which allows to treat the case of a class of semilinear equations following the (fixed-point) method of [4,5] (generalized in [6]). Carleman estimates also have other applications in analysis, e.g., uniqueness results, inverse problems.

The Carleman estimates derived here for the operators $\partial_{t} \pm \partial_{x}\left(c \partial_{x}\right)$ are obtained through a limiting process from the Carleman estimates associated for $\partial_{t} \pm \partial_{x}\left(c_{\varepsilon} \partial_{x}\right)$, for $c_{\varepsilon}$ piecewise regular converging to $c$ in $L^{\infty}(\Omega)$. The main issue in this limiting process is to keep both the weight functions and constants in the Carleman estimate under control. Controllability results of this type can be motivated by problems from biology for example. Note that the approximation of the $B V$ coefficient $c$ by some piecewise regular coefficient $c_{\varepsilon}$ is closely related to numerical methods.

\section{Carleman estimates in the case of a $B V$ coefficient}

We first consider a piecewise $\mathscr{C}^{1}$ diffusion coefficient $c$ with a finite number of singularities. Here, we shall assume that $0=a_{0}<a_{1}<\cdots<a_{n}=1$ and $c_{\mid\left[a_{i}, a_{i+1}\right]} \in \mathscr{C}^{1}\left(\left[a_{i}, a_{i+1}\right]\right), i=0, \ldots, n-1$. We shall use the following notations $S=\left\{a_{1}, \ldots, a_{n-1}\right\}, \Omega^{\prime}=\Omega \backslash S$, and $Q^{\prime} \stackrel{\left(a, a_{t+1}\right\}}{=}(0, T) \times \Omega^{\prime}$.

Lemma 2.1 ([2]) There exists a function $\widetilde{\beta} \in \mathscr{C}(\Omega)$ such that $\widetilde{\beta}_{\left[a_{i}, a_{i+1}\right]} \in \mathscr{C}^{2}\left(\left[a_{i}, a_{i+1}\right]\right), i=0, \ldots, n-1$, satisfying $\widetilde{\beta}>0$ in $\Omega, \widetilde{\beta}(1)=0,\left(\widetilde{\beta}_{\left|a_{i}, a_{i+1}\right|}\right)^{\prime}<0, i \in\{0, \ldots, n-1\}$, and the function $\widetilde{\beta}$ satisfies the following trace properties, for some $\alpha>0$,

$$
\left(A_{i} u, u\right) \geq \alpha|u|^{2}, \quad \text { with } A_{i}=\left(\begin{array}{ll}
{\left[\widetilde{\beta^{\prime}}\right]_{a_{i}}} & \widetilde{\beta^{\prime}}\left(a_{i}^{+}\right)\left[\widetilde{\beta^{\prime}}\right]_{a_{i}} \\
\left.\widetilde{\beta^{\prime}}\left(a_{i}^{+}\right)\left[c \widetilde{\beta^{\prime}}\right]_{a_{i}}{\widetilde{\beta^{\prime}}}^{\prime}\left(a_{i}^{+}\right)\left[\widetilde{c \beta^{\prime}}\right]_{a_{i}}^{2}+\left[c^{2} \widetilde{\beta^{\prime}}\right)^{3}\right]_{a_{i}}
\end{array}\right), \quad u \in \mathbb{R}^{2},
$$

for $i=1, \ldots, n-1$, and where $[\rho]_{x}=\rho\left(x^{+}\right)-\rho\left(x^{-}\right)$for $x \in(0,1)$.

Choosing a function $\widetilde{\beta}$, as in the previous lemma, we introduce $\beta=\widetilde{\beta}+K$ with $K=m\|\widetilde{\beta}\|_{\infty}$ and $m>1$. For $\lambda>0$ and $t \in(0, T)$, we define the following weight functions,

$$
\varphi(t, x)=\frac{e^{\lambda \beta(x)}}{t(T-t)}, \quad \eta(t, x)=\frac{e^{\lambda \bar{\beta}}-e^{\lambda \beta(x)}}{t(T-t)},
$$

with $\bar{\beta}=2 m\|\widetilde{\beta}\|_{\infty}$ (see $\left.[11,12]\right)$. We introduce

$$
\begin{aligned}
\aleph=\left\{q \in \mathscr{C}(Q, \mathbb{R}) ; q_{\mid[0, T] \times\left[a_{i} a_{i+1}\right]} \in \mathscr{C}^{2}\left([0, T] \times\left[a_{i}, a_{i+1}\right]\right), i=0, \ldots, n-1, q_{\mid \Sigma}=0,\right. \\
\left.\quad \text { and } q \text { satisfies, } q\left(t, a_{i}^{-}\right)=q\left(t, a_{i}^{+}\right), c\left(a_{i}^{-}\right) \partial_{x} q\left(t, a_{i}^{-}\right)=c\left(a_{i}^{+}\right) \partial_{x} q\left(t, a_{i}^{+}\right), i=1, \ldots, n-1, \text { for all } t \in(0, T)\right\} .
\end{aligned}
$$

With the function $\widetilde{\beta}$ satisfying the properties of Lemma 2.1, we obtain the following Carleman estimate.

Theorem 2.2 ([2]) There exist $\lambda_{1}>0, s_{1}=\left(T+T^{2}\right) \widetilde{s}_{1}>0$ and $C>0$ so that the following estimate holds, for $s \geq s_{1}, \lambda \geq \lambda_{1}$ and for all $q \in \boldsymbol{\aleph}$, 


$$
\begin{array}{r}
s^{-1} \iint_{Q} e^{-2 s \eta} \varphi^{-1}\left(\left|\partial_{t} q\right|^{2}+\left|\partial_{x}\left(c \partial_{x} q\right)\right|^{2}\right) d x d t+s \lambda^{2} \iint_{Q} e^{-2 s \eta} \varphi\left|\partial_{x} q\right|^{2} d x d t+s^{3} \lambda^{4} \iint_{Q} e^{-2 s \eta} \varphi^{3}|q|^{2} d x d t \\
\leq C\left[s \lambda \int_{0}^{T} \varphi(t, 0) e^{-2 s \eta(t, 0)}\left|\partial_{x} q\right|^{2}(t, 0) d t+\iint_{Q} e^{-2 s \eta}\left|\partial_{t} q \pm \partial_{x}\left(c \partial_{x} q\right)\right|^{2} d x d t\right] .
\end{array}
$$

We now consider a diffusion coefficient $c \in B V(\Omega)$, with $0<c_{\min } \leq c \leq c_{\max }$. We denote the total variation of $c$ on $(0,1)$ by $\vartheta:=V_{0}^{1}(c)$. Let $\varepsilon>0$. There exists a piecewise-constant function $c_{\varepsilon}$ such that $\left\|c-c_{\varepsilon}\right\|_{L^{\infty}(\Omega)} \leq \varepsilon$, $V_{0}^{1}\left(c_{\varepsilon}\right) \leq \vartheta$ (see e.g. [13]).

We denote by $a_{1}, \ldots, a_{k}$ the points of discontinuity of $c_{\varepsilon}$. We then have $\sum_{i=1}^{k}\left|c_{\varepsilon}\left(a_{i}^{+}\right)-c_{\varepsilon}\left(a_{i}^{-}\right)\right| \leq \vartheta$. Let $Y_{i}=$ $c_{\varepsilon}\left(a_{i}^{+}\right) / c_{\varepsilon}\left(a_{i}^{-}\right)$and $X_{i}, i=1, \ldots, k$, be defined by $X_{i}=f\left(Y_{i}\right)$, with $f(s)=s$ if $s \geq 1$ and $f(s)=2-s$ if $s<1$. We define the piecewise-constant function $\gamma_{\varepsilon}$ as $\gamma_{\varepsilon}(x):=\gamma(1) \prod_{x<a_{j}} X_{j}$, for $x \notin\left\{a_{1}, \ldots, a_{k}\right\}$, for some fixed $\gamma(1)<0$. We set the function $\widetilde{\beta}_{\varepsilon}(x):=\int_{1}^{x} \gamma_{\varepsilon}(y) d y$, which satisfies the properties listed in Lemma 2.1 by the jump conditions imposed on $\gamma_{\varepsilon}=\widetilde{\beta}_{\varepsilon}^{\prime}$ at $a_{1}, \ldots, a_{k}$. Concerning the total variation of the functions $\gamma_{\varepsilon}$ we have the following lemma. Lemma 2.3 There exist $K>0$ and $\varepsilon_{0}>0$ that solely depend on the diffusion coefficient $c \in B V(\Omega)$ such that, for all $0<\varepsilon \leq \varepsilon_{0}, V_{0}^{1}\left(\gamma_{\varepsilon}\right) \leq K|\gamma(1)|$.

By Helly's theorem [3,13], up to a subsequence, the functions $\gamma_{\varepsilon}$ converge everywhere to a function $\gamma$ as $\varepsilon$ goes to 0 . Moreover, this function satisfies $V_{0}^{1}(\gamma) \leq K|\gamma(1)|$. By dominated convergence, the associated functions $\widetilde{\beta}_{\varepsilon}$ converge everywhere to the continuous function $\widetilde{\beta}(x):=\int_{1}^{x} \gamma(y) d y$.

Let $a$ be any point of discontinuity of $c_{\varepsilon}$. As above, we set $Y=\frac{c_{\varepsilon}\left(a^{+}\right)}{c_{\varepsilon}\left(a^{-}\right)}$and define the matrix $A$ as in Lemma 2.1. Then we have the following crucial lemma.

Lemma 2.4 The eigenvalues $v_{1}, v_{2}$ of the matrix A satisfy $v_{i} \geq C|Y-1|, i=1,2$, with $C$ uniform w.r.t. $\varepsilon$ and the considered singularity of $c_{\varepsilon}$.

With $\widetilde{\beta}_{\varepsilon}$ and $\widetilde{\beta}$, we define weight functions according to (3). With a detailed inspection of the proof of the Carleman estimate in the case of a piecewise $\mathscr{C}^{1}$ coefficient [2], the previous lemma yields

Proposition 2.5 The constant $C$ on the r.h.s. of the Carleman estimate (4) for $\partial_{t} \pm \partial_{x}\left(c_{\varepsilon} \partial_{x}\right)$ and the constants $s_{1}$ and $\lambda_{1}$ can be chosen uniformly w.r.t. $\varepsilon$ for $0<\varepsilon \leq \varepsilon_{0}$, with $\varepsilon_{0}$ sufficiently small.

The previous results show that both the weight functions and the constants in the Carleman estimate remain 'well behaved', as $\varepsilon$ goes to zero. We consider $q$ and $q_{\varepsilon}$ (weak) solutions to

$$
\left\{\begin{array} { l l } 
{ \partial _ { t } q \pm \partial _ { x } ( c \partial _ { x } q ) = f } & { \text { in } Q , } \\
{ q = 0 } & { \text { on } \Sigma , } \\
{ q ( T , x ) = q _ { 0 } ( x ) ( \text { resp. } q ( 0 , x ) = q _ { 0 } ( x ) ) } & { \text { in } \Omega . }
\end{array} \left\{\begin{array}{ll}
\partial_{t} q_{\varepsilon} \pm \partial_{x}\left(c_{\varepsilon} \partial_{x} q_{\varepsilon}\right)=f & \text { in } Q, \\
q_{\varepsilon}=0 & \text { on } \Sigma, \\
q_{\varepsilon}(T, x)=q_{0, \varepsilon}(x)\left(\text { resp. } q_{\varepsilon}(0, x)=q_{0, \varepsilon}(x)\right) & \text { in } \Omega,
\end{array}\right.\right.
$$

with the initial data $q_{0}$ and $q_{0, \varepsilon}$ given by $\partial_{x}\left(c \partial_{x} q_{0}\right)=\mu$ and $\partial_{x}\left(c_{\varepsilon} \partial_{x} q_{0, \varepsilon}\right)=\mu$, with the same $\mu$, where $\mu$ and $f$ are sufficiently regular. This choice of initial data, yields the convergence of $q_{0, \varepsilon}$ to $q_{0}$ in $H_{0}^{1}(\Omega)$ as $\varepsilon$ goes to zero. Since $\partial_{t} q_{\varepsilon}, q_{\varepsilon}, \partial_{x} q_{\varepsilon}$ and $\partial_{x}\left(c \partial_{x} q_{\varepsilon}\right)$ converge to $\partial_{t} q, q, \partial_{x} q$ and $\partial_{x}\left(c \partial_{x} q\right)$ in $L^{2}$ norm, the previous results show that the Carleman estimate for $q_{\varepsilon}$ and the operators $\partial_{t} \pm \partial_{x}\left(c_{\varepsilon} \partial_{x}\right)$ yield a similar estimate for $q$ and the operators $\partial_{t} \pm \partial_{x}\left(c \partial_{x}\right)$ with the same constants, as $\varepsilon$ goes to zero. We can now relax the assumptions on $\mu$ and $f$. We have thus obtained the following theorem.

Theorem 2.6 Let $c \in B V(\Omega)$ with $0<c_{\min } \leq c \leq c_{\max }$. There exists $\lambda_{1}>0, s_{1}=\left(T+T^{2}\right) \widetilde{s}_{1}>0$ and $C>0$ so that the Carleman estimate (4) holds for $s \geq s_{1}, \lambda \geq \lambda_{1}$ and for all $q$ (weak) solution to $\partial_{t} q \pm \partial_{x}\left(c \partial_{x} q\right)=f$ in $Q, q=0$ on $\Sigma, q(T, x)=q_{0}(x)\left(\right.$ resp. $\left.q(0, x)=q_{0}(x)\right)$ in $\Omega$, with $q_{0} \in L^{2}(\Omega)$ and $f \in L^{2}(Q)$.

In the case where $O \Subset \Omega$, with $O$ a non-empty open set and $c$ is of class $\mathscr{C}^{1}$ in $O$, we obtain Carleman estimates, for the operators $\partial_{t} \pm \partial_{x}\left(c \partial_{x}\right)$, with an interior 'observation' on $(0, T) \times O$ i.e. with the term 


$$
s^{3} \lambda^{4} \iint_{(0, T) \times O} e^{-2 s \eta} \varphi^{3}|q|^{2} d x d t
$$

replacing the time integral on the r.h.s. of (4).

In the case of an interior 'observation', the proof is similar to the previous one. The function $\widetilde{\beta}_{\varepsilon}$ is constructed on both sides of $O$, through its derivative, and the regularity of $c$ in $O$ allows to smoothly connect the two parts of the function, with some convergence in $\mathscr{C}^{2}(\bar{O})$ as $\varepsilon$ goes to zero. As above, we then pass to the limit in a Carleman estimate for $\partial_{t} \pm \partial_{x}\left(c_{\varepsilon} \partial_{x}\right)$, with $c_{\varepsilon}$ piecewise $\mathscr{C}^{1}$, with an interior 'observation', proven in [2].

\section{A Carleman estimate for the heat equation with a r.h.s. in $L^{2}\left(0, T, H^{-1}(\Omega)\right)$}

Following [14], we obtain a Carleman estimate for $\partial_{t} q \pm \partial_{x}\left(c \partial_{x} q\right)=f$ if $f \in H^{-1}$. We set

$$
\widetilde{\boldsymbol{\kappa}}_{ \pm} \quad=\quad\left\{q \in \mathscr{C}\left([0, T], H_{0}^{1}(\Omega)\right) ; q(t) \in D(A) \forall t \in[0, T], \partial_{t} q \pm \partial_{x}\left(c \partial_{x} q\right)=F_{0}+\partial_{x} F_{1}, F_{0}, F_{1} \in L^{2}(Q)\right\} .
$$

Theorem 3.1 Let $O \Subset \Omega$ be a non-empty open set and $c \in B V(\Omega)$ with $0<c_{\min } \leq c \leq c_{\max }$ and $c$ of class $\mathscr{C}^{1}$ in $O$. There exists $\lambda_{2}=\lambda_{2}(O, c)>0, s_{2}=\left(T+T^{2}\right) s_{2}(O, c)>0$ and a positive constant $C=C(O, c)$ so that the following estimate holds, for $s \geq s_{2}, \lambda \geq \lambda_{2}$ and for all $q \in \widetilde{\boldsymbol{\aleph}}_{ \pm}$,

$$
\begin{array}{rl}
s \lambda^{2} \iint_{Q} e^{-2 s \eta} \varphi\left|\partial_{x} q\right|^{2} d x d t+s^{3} \lambda^{4} \iint_{Q} e^{-2 s \eta} \varphi^{3}|q|^{2} & d x d t \leq C\left[s^{3} \lambda^{4} \iint_{(0, T) \times O} e^{-2 s \eta} \varphi^{3}|q|^{2} d x d t\right. \\
& \left.+\iint_{Q} e^{-2 s \eta}\left|F_{0}\right|^{2} d x d t+s^{2} \lambda^{2} \iint_{Q} e^{-2 s \eta} \varphi^{2}\left|F_{1}\right|^{2} d x d t\right] .
\end{array}
$$

\section{Controllability results}

The Carleman estimate (5) proven in the previous section allows to give observability estimates that yield results of controllability to the trajectories for classes of semilinear heat equations. We let $\omega \Subset \Omega$ be a non-empty open set and $c \in B V(\Omega)$ with $0<c_{\min } \leq c \leq c_{\max }$ and $c$ of class $\mathscr{C}^{1}$ in some non-empty open subset of $\omega$. We let $a$ and $b$ be in $L^{\infty}(Q)$ and $q_{T} \in L^{2}(\Omega)$. From Carleman estimate (5) we obtain the following lemma.

Lemma 4.1 The solution $q$ to $-\partial_{t} q-\partial_{x}\left(c \partial_{x} q\right)+a q-\partial_{x}(b q)=0$ in $Q, q=0$ on $\Sigma$, and $q(T)=q_{T}$ satisfies $\|q(0)\|_{L^{2}(\Omega)}^{2} \leq e^{C H}\left(\iint_{(0, T) \times \omega}|q| d x d t\right)^{2}$, where $H=H\left(T,\|a\|_{\infty},\|b\|_{\infty}\right)=1+\frac{1}{T}+T+\left(T+T^{1 / 2}\right)\|a\|_{\infty}+\|a\|_{\infty}^{2 / 3}+(1+T)\|b\|_{\infty}^{2}$.

Since the coefficient $c$ is $\mathscr{C}^{1}$ in some open subset of $\omega$, the proof of [6, Theorem 2.5, Lemma 2.5] can be adapted. See also [12, Proposition 4.2, Lemma 4.3]. Such an observability estimate yields the null controllability of the semilinear parabolic system (1), as well as the following system

$$
\begin{cases}\partial_{t} y-\partial_{x}\left(c \partial_{x} y\right)+\mathscr{G}\left(y, \partial_{x} y\right)=1_{\omega} v & \text { in } Q, \\ y(t, .)=0 & \text { on } \Sigma, \\ y(0, x)=y_{0}(x) & \text { in } \Omega,\end{cases}
$$

where $\mathscr{G}: \mathbb{R}^{2} \rightarrow \mathbb{R}$ is locally Lipschitz and $\mathscr{G}(0,0)=0$, which implies that $\mathscr{G}(x, y)=x g(x, y)+y G(x, y)$, with $g$ and $G$ in $L_{\mathrm{loc}}^{\infty}(\mathbb{R})$.

Theorem 4.2 Let $T>0$. Let $c \in B V(\Omega)$ with $0<c_{\min } \leq c \leq c_{\max }$.

(i) Local null controllability: There exists $\varepsilon>0$ such that for all $y_{0}$ in $L^{2}(\Omega)$ with $\left\|y_{0}\right\|_{L^{2}(\Omega)} \leq \varepsilon$, there exists a control $v \in L^{\infty}(0, T)$ such that the solution to (1) satisfies $y(T)=0$. 
(ii) Global null controllability: Let $\mathscr{G}$ satisfy in addition

$$
\lim _{|(x, y)| \rightarrow \infty} \frac{|g(x, y)|}{\ln ^{3 / 2}(1+|x|+|y|)}=0 \quad \text { and } \lim _{|(x, y)| \rightarrow \infty} \frac{|G(x, y)|}{\ln ^{1 / 2}(1+|x|+|y|)}=0 .
$$

For all $y_{0} \in L^{2}(\Omega)$, there exists $v \in L^{\infty}(0, T)$ such that the solution to (1) satisfies $y(T)=0$.

We have similar results for system (6) with a non-empty open subset $\omega \Subset \Omega$, and $v \in L^{\infty}((0, T) \times \omega)$, assuming that c of class $\mathscr{C}^{1}$ in some non-empty open subset of $\omega$.

The proof is based on a fixed-point argument and is along the same lines as that in [6] and originates from [4,5]. Note that as usual, $y(T)=y^{*}(T)$ can replace $y(T)=0$ in the previous statements, where $y^{*}$ is any trajectory defined in $[0, T]$ of system (1), corresponding to some initial data $y_{0}^{*} \in L^{2}(\Omega)$ and any $v^{*}$ in $L^{\infty}(0, T)\left(L^{\infty}((0, T) \times \omega)\right.$ in the case of an interior control). For the local controllability result, one has to assume $\left\|y_{0}-y_{0}^{*}\right\|_{L^{2}(\Omega)} \leq \varepsilon$, with $\varepsilon$ sufficiently small.

\section{Acknowledgements}

The author wishes to thank A. Benabdallah and Y. Dermenjian for numerous discussions on the subject of this article.

\section{References}

[1] A. Benabdallah, Y. Dermenjian, J. Le Rousseau, Carleman estimates for the one-dimensional heat equation with a discontinuous coefficient and applications, Comptes Rendus Mécanique 334 (2006) 582-586.

[2] A. Benabdallah, Y. Dermenjian, J. Le Rousseau, Carleman estimates for the one-dimensional heat equation with a discontinuous coefficient and applications to controllability and an inverse problem, Preprint: LATP, Université d'Aix-Marseille I, www . cmi . univ-mrs. fr/ jlerous/publications.html, 2006.

[3] A. Kolmogorov, S.V. Fomin, Eléments de la théorie des fonctions et de l'analyse fonctionnelle, Editions MIR, 1974.

[4] V. Barbu, Exact controllability of the superlinear heat equation, Appl. Math. Optim. 42 (2000) $73-89$.

[5] E. Fernández-Cara, E. Zuazua, Null and approximate controllability for weakly blowing up semilinear heat equations, Ann. Inst. H. Poincaré, Analyse non lin. 17 (2000) 583-616.

[6] A. Doubova, E. Fernandez-Cara, M. Gonzales-Burgos, E. Zuazua, On the controllability of parabolic systems with a nonlinear term involving the state and the gradient, SIAM J. Control Optim. 41 (2002) 798-819.

[7] E. Fernández-Cara, E. Zuazua, On the null controllability of the one-dimensional heat equation with $B V$ coefficients, Comput. Appl. Math. 21 (2002) 167-190.

[8] D. L. Russell, A unified boundary controllability theory for hyperbolic and parabolic partial differential equations, Studies in Appl. Math., 52 (1973) 189-221.

[9] G. Alessandrini, L. Escauriaza, Null-controllability of one-dimensional parabolic equations, ESAIM Control Optim. Calc. Var., accepted for publication.

[10] G. Lebeau, L. Robbiano, Contrôle exact de l'équation de la chaleur, Comm. Part. Diff. Eqs. 20 (1995) $335-356$.

[11] A. Fursikov, O. Yu. Imanuvilov, Controllability of evolution equations, Lecture notes 34, Seoul National University, Korea, 1996

[12] A. Doubova, A. Osses, J.-P. Puel, Exact controllability to trajectories for semilinear heat equations with discontinuous diffusion coefficients, ESAIM: Control Optim. Calc. Var. 8 (2002) 621-661.

[13] A. Bressan, Hyperbolic Systems of Conservation Laws: The One Dimensional Cauchy Problem, Oxford University Press, 2000.

[14] O. Yu. Imanuvilov, M. Yamamoto, Carleman estimate for a parabolic equation in a Sobolev space of negative order and its applications, Lecture Notes in Pure and Applied Mathematics 218, 113-137, Dekker, New York, 2001. 